\title{
The Effect of Communication and Vehicle Properties on the Search Performance of a Swarm of Unmanned Aerial Vehicles
}

Jenna E. Newcomb

Brigham Young University, jennewcomb178@gmail.com

Andrew Ning

Brigham Young University, aning@byu.edu

Follow this and additional works at: https://scholarsarchive.byu.edu/facpub

Part of the Military Vehicles Commons

\section{Original Publication Citation}

Newcomb, J. E., and Ning, A., "The Effect of Communication and Vehicle Properties on the Search Performance of a Swarm of Unmanned Aerial Vehicles," AIAA Scitech 2019 Forum, San Diego, CA, Jan. 2019. doi:10.2514/6.2019-1573

\section{BYU ScholarsArchive Citation}

Newcomb, Jenna E. and Ning, Andrew, "The Effect of Communication and Vehicle Properties on the Search Performance of a Swarm of Unmanned Aerial Vehicles" (2019). Faculty Publications. 2979. https://scholarsarchive.byu.edu/facpub/2979 


\title{
The Effect of Communication and Vehicle Properties on the Search Performance of a Swarm of Unmanned Aerial Vehicles
}

\author{
Jenna E. Newcomb* and Andrew Ning ${ }^{\dagger}$ \\ Brigham Young University, Provo, UT,84602
}

\begin{abstract}
An unmanned aerial vehicle (UAV) swarm allows for a more time-efficient method of searching a specified area than a single UAV or piloted plane. There are a variety of factors that affect how well an area is surveyed. We specifically analyzed the effect both vehicle properties and communication had on the swarm search performance. We used non-dimensionalization to examine the effect vehicle properties had on search performance so the results can be applied to any domain size with any number and type of vehicle. We found that even if vehicles could only sense $10 \%$ of the grid area at any given time step, $92 \%$ of the grid could still be searched by using a vehicle with good maneuverability and endurance. Conversely, if the chosen vehicle had poor maneuverability $92 \%$ of the grid could still be searched by keeping the endurance high and increasing the amount of area the vehicles could sense at one time to $70 \%$ of the domain area. In addition, we also examined four communication cases with different amounts of information regarding vehicle location. We found communication increased search performance by at least $6.3 \%$. However, increasing the amount of information only changed the performance by $2.3 \%$.
\end{abstract}

Flight path angle

Nomenclature

$\phi \quad$ Roll angle

$\sigma^{2} \quad$ Variance of grid value distribution

$d_{i} \quad$ Distance between $i^{\text {th }}$ cell and closest vehicle

$g \quad$ Acceleration due to gravity

$J \quad$ Potential reward for planned path

$L \quad$ Domain length

$l_{c} \quad$ Length of grid cell side

$N_{g} \quad$ Total number of grid cells

$N_{v} \quad$ Number of vehicles

$p_{e} \quad$ Inertial east position of the vehicle

$p_{n} \quad$ Inertial north position of the vehicle

$r_{m} \quad$ Maximum value of grid cell

$R_{S} \quad$ Sensing radius

$R_{t} \quad$ Turning radius

$R_{c} \quad$ Communication radius

$r_{i} \quad$ Value of $i^{t h}$ cell

* Masters Student, Department of Mechanical Engineering, Brigham Young University, AIAA Student Member

${ }^{\dagger}$ Assistant Professor, Department of Mechanical Engineering, Brigham Young University, AIAA Senior Member. 
$t \quad$ Length of time step

$V_{a} \quad$ Airspeed

$V_{c r} \quad$ Cruise velocity of vehicle

$V_{g} \quad$ Ground speed

$V_{s} \quad$ Stall speed of vehicle

\section{Introduction}

The aerial surveillance market is estimated to grow to be worth $\$ 5.81$ billion by 2023 * Unmanned aerial vehicles (UAVs) provide many opportunities to reduce manual labor. Some uses of aerial reconnaissance, such as locating an object or person, will require the use of many UAVs. However, the use of many vehicles requires coordination, and the use of shared information that can require a significant amount of bandwidth and expensive calculations. For this reason, information should be limited to that which is necessary for mission success. Although there are many path planning methods in literature, little work has been compiled analyzing what amount of information is necessary. We sought to address this issue and determine how the amount of information shared between vehicles affects the swarm search performance. Another issue that we felt was inadequately studied was the effect certain vehicle properties had on searching missions. Others have created trajectory optimization methods subject to vehicle operational constraints. However, we varied these constraints to determine which properties significantly affect search completion. Therefore, we can use these results to choose or design a vehicle that is optimal for the reconnaissance mission.

When operating simultaneously, aerial vehicles must communicate to remain dispersed and accomplish missions. Without communication, an UAV swarm will operate inefficiently. Researchers have explored UAV swarm path planning and mission assignment [1,-5], and how vehicles communicate [6, 7]. However, little has been done to determine the minimum amount of information sharing required for mission success. Waharte and Rigoni compared the effect sharing data has on a search and rescue mission through analyzing the results of different optimization methods [8]. Their goal was to minimize the time required for the UAVs to locate a target. Partially observable Markov decision process based, potential-based, and greedy processes were used for the search algorithms. The study concluded that the partially observable Markov decision process and look ahead methods were the most effective when it came to reducing the time to find the target. These results showed that the most suitable paths came from methods in which vehicles shared and used information which potentially showed the location of the target. Although these methods were effective they were computationally expensive. Waharte and Rigoni proved that partially observable Markov decision process based optimization was effective when the number of states was limited. This study was primarily focused on the effectiveness of the optimization methods. Some methods shared data and others did not. Since communication was not the only varying factor, it is not known how much the communication contributed to increasing the swarm's performance.

Krieger and Billeter used a group of robots to gather food items for energy [9]. They utilized a decentralized task distribution method based on insect swarm behavior. They performed the experiment both with no coordination and with a simple coordination approach in which robots could guide other members to the discovered food source. In the end, they concluded that coordinated communication increased total group performance by 13 percent. Arkin, Balch, and Nitz showed that communication can increase performance and productivity in a robotic swarm [10]. They assessed task completion for two scenarios: the first with no communication, and the second with a collective memory. If a robot was unable to locate a goal, it used the collective memory to find a robot that had found a goal. They measured performance as the average distance traveled by the robots. The results showed that communication does not have a significant effect on total distance. However, it was also shown that the number of return steps decreased, showing that communication allowed more efficient behavior. Trianni and Dorigo used common behavior strategies found in insect colonies to create swarm algorithms [11]. They analyzed both direct and indirect communication as well as direct interactions for a group of s-bots attempting to avoid holes in a specified area. They concluded that using direct communication resulted in far fewer hole encounters. This study demonstrated that for a group of robots with a common goal, communication is necessary. These studies do focus on the effect of communication alone, however, they are all-or-nothing approaches: the robots performed either with, or without, communication. There was no testing to

\footnotetext{
*ttps://www.marketsandmarkets.com/PressReleases/airborne-surveillance.asp
} 
determine how much information needed to be shared. In contrast, we tested a range of communication amounts to determine the increase in group performance. As a result, we likewise know that communication does improve search performance and how much different amounts improve performance. Therefore, we can not only show the importance of information sharing, but we can limit the information to that which is necessary.

We combined surveillance performance with vehicle design to discover how vehicle parameters affected the mission outcome. Many people have created trajectory optimization methods subject to dynamic vehicle constraints. Sujit and Ghose combined path optimization with vehicle endurance and used endurance as a constraint [12]. They only allowed the UAVs to travel a specified number of steps before needing to return to refuel. Blasi, Barbato, and Mattei used particle swarm optimization to optimize flight paths subject to specific operational constraints including minimum turning radius, range and endurance, maximum climbing rate, maximum payload capacity, and maximum and minimum speed [13]. These studies sought to find a way to optimize paths with vehicle operational constraints, whereas we wanted to know how changing these operational properties affect search performance. In essence, we were not concerned with how to optimize paths constrained to a certain vehicle but how to design or choose a vehicle which is optimal for a given search mission.

Nigma, Bieniawsi, Kroo, and Vian account for dynamic constraints in their proposed control policy [14]. Using a 3-DOF motion model, they analyzed how dynamics affected the performance of the UAVs. Their performance metric was the cell age based on when the cell was last explored. Their analysis showed that when using the Actual Distance Policy, where the actual distances reflect the dynamic constraints, the maximum cell ages increased from 79 to 106.6 when increasing the turning radius from 1.67 to $5 \mathrm{~m}$ and increasing $C_{L m a x}$ from 1.03 to 1.67 . Additionally, they use the a health monitoring policy to determine when UAVs should refuel based on the vehicles' endurance. Nigma, Bieniawsi, Kroo, and Vian looked into how dynamics affected search performance, but their primary motivation was to compare the Actual Distance Policy to the Euclidean Distance Policy under dynamic constraints. We non-dimensionalized the operational properties to find the effect they had on mission performance. Our end goal was to know what operational properties affected the search performance most significantly.

The majority of these studies focused on path planning optimization. The studies that focused on the benefits of communication used all or nothing approaches. Instead, we analyzed the results of the vehicle swarm performance for a variety of communication.

The research that took into account vehicle design ([12], [13], [14]) created optimization methods which used vehicle properties as constraints. Instead, we varied the vehicle and domain properties across a wide range to determine the effect vehicle properties had on search performance. We wanted to know what properties a vehicle needed to maximize performance for a certain mission. Additionally, through the use of non-dimensionalization we can apply these results to a search mission with any domain size and number of vehicles.

\section{Methods}

Our end goal was to determine what swarm properties had the most effect on surveillance completion. This task was broken up into two parts: finding the relationships between vehicle properties and search performance and discovering to what extent the amount of information shared between swarm members affected how much of the domain was searched. Our objective was to maximize the searched area within a domain. The grid space was divided evenly into 900 grid cells $(30 \times 30)$. Fig. 1 shows the grid area and the initial starting location of the UAVs. The UAVs are the blue circles and the lines are the current orientation of each UAV. Every optimization had the same initial conditions and vehicles began in the same evenly spaced locations and orientations. To simplify analysis, it was assumed there were no outside factors including wind or environmental obstacles. 


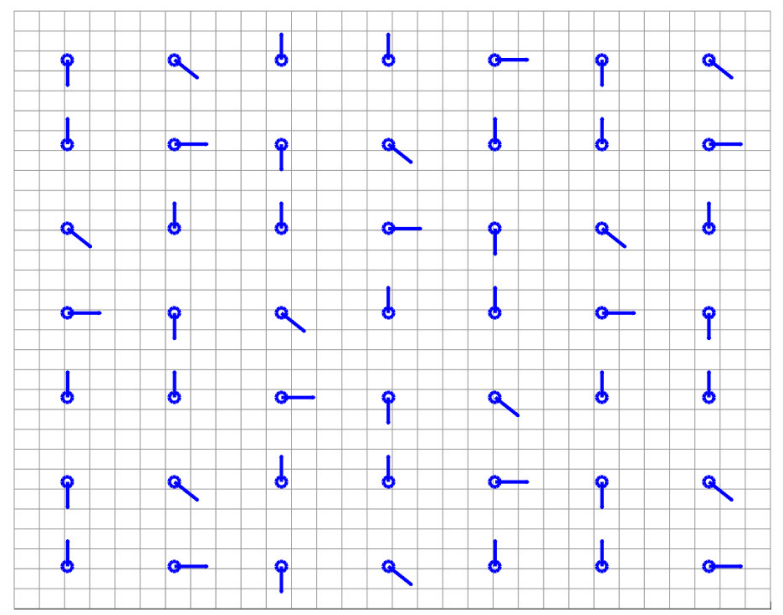

Fig. 1 The 49 vehicles began in the same location and in the same orientation for every simulation. The vehicles were distributed in seven rows and columns. The small squares are the grid cells which update every time step based their value.

The grid cells contained an associated reward value, $r_{i}$, which began at a minimum value of zero. A value of zero meant the cell had not yet been seen and vehicles should have proceeded to that cell to increase its value. Rather than provide a constant reward value across the cell, we used a Gaussian distribution to allow the reward to vary continuously and permit the use of gradient-based optimization. Fig. 2 shows the value associated with the distance between a vehicle and a grid center. The realized reward was the largest value obtained in each cell. See Eq. 1 for how we updated the grid value of cell $i$.

$$
r_{i}=e^{-\frac{d_{i}^{2}}{2 \sigma^{2}}}
$$

The distance between a vehicle and a particular cell, $i$, is given by $d_{i}$. The value of $\sigma$ was set to the vehicle's sensing radius divided by three because we wanted most of the reward contained within the sensing radius. In addition, the cell reward increased significantly as the vehicle approached the cell center.

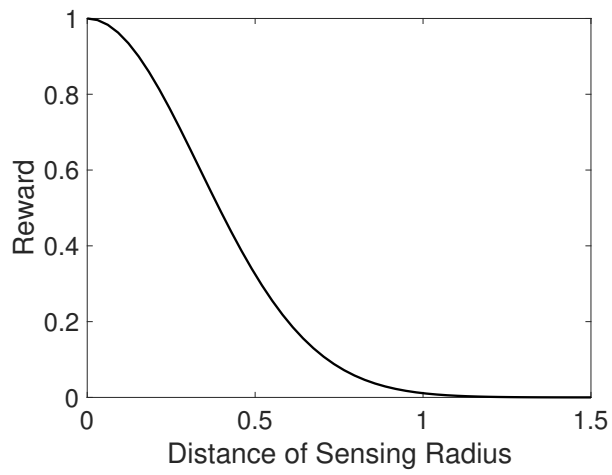

Fig. 2 The reward distribution as a function of the distance divided by the sensing radius.

We used gradient-based optimization to maximize the reward by changing the future planned roll angles. The reward was the summed predicted values of all the grid cells from the potential path. The vehicle motion was based on a coordinated turn approach where an ODE solver determined the heading angle, north location, and east location of the vehicle after every roll angle. 
The vehicle search performance is given by Eq. 2. It was the fraction of the grid that was seen after the simulation was completed.

$$
P=\frac{\sum_{i=1}^{N_{g}} r_{i}}{r_{m} N_{g}}
$$

where $r_{i}$ is the $i t h$ cell's value, $r_{m}$ is the maximum value of the grid cell, and $N_{g}$ is the number of grid cells. The cell value is summed across every cell then divided by $r_{m}$ times $N_{g}$, which is the maximum attainable reward for the entire grid. In our simulation, $r_{m}$ was equal to one.

\section{A. Vehicle Model}

We modeled the UAVs as point masses using a simple coordinated-turn approach. The turning radius is given by Eq. 3 To simplify analysis, we assumed the sideslip angle, $\beta$, is zero and that there was no wind. Therefore, the equation for the change in yaw, $\dot{\psi}$, is given by Eq. 4 . The inertial north and east of the vehicle are then given by Eq. 5 and 6 respectively.

$$
\begin{aligned}
R_{t} & =\frac{V_{g}^{2} \cos \gamma}{g \tan \phi} \\
\dot{\psi} & =\frac{g}{V_{a}} \tan \phi \\
\dot{p_{n}} & =V_{c r} \cos \psi \\
\dot{p_{e}} & =V_{c r} \sin \psi
\end{aligned}
$$

\section{B. Nondimensionalization}

Since search missions vary by size, duration, and number of vehicles, we wanted to find the relationships between vehicle properties and search performance that can apply to all types of search missions. In addition, determining the effect of a mission parameter on search performance can be difficult to find because the performance also depends on other parameters. Because of this, we non-dimensionalized the properties and analyzed how the search performance, given by Eq. 2, changed with varying the non-dimensional numbers. This generalized the results and gave us insight into which parameters are critical as opposed to parameters that may not have an effect on performance and can be relaxed. The properties included turning radius, communication radius, sensing radius, cruise velocity, flight time, number of vehicles, and maximum roll angle. We assumed no communication occurred unless a vehicle entered the communication radius of another vehicle. If this occurred, the vehicles communicated their future predicted paths. We had six main non-dimensional parameters.

The first parameter is the endurance ratio and is defined as:

$$
\frac{N_{v} V_{c r} T}{L}
$$

This ratio represents the distance the vehicles can cover relative to the length of the domain. It is based on the vehicles' endurance given by $T$. A graphic of this number for one vehicle is shown in Fig. 3. The longer the vehicles can fly, the better the search performance. The ratio demonstrates how suitable the number of aircraft is based on the vehicles' endurance and cruise velocity for a particular domain size. Therefore, large domain sizes will require either many vehicles or vehicles with high endurance. To test a wide range of ratios we ranged the endurance ratio from 35.0 to 175.0 with 5 equally spaced points. 


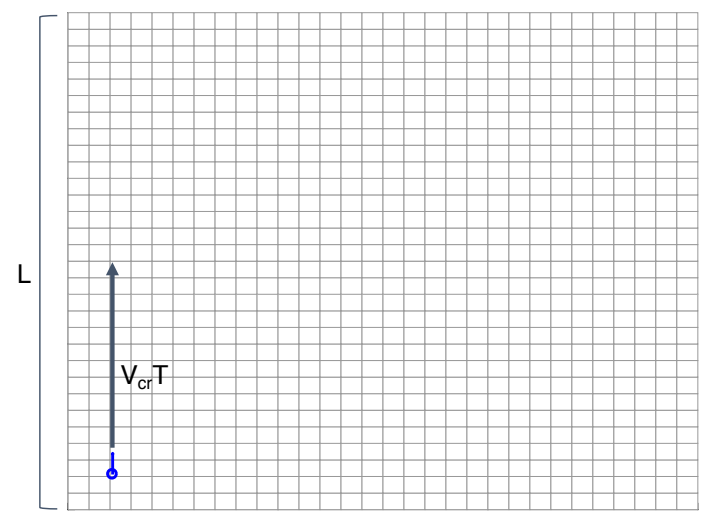

Fig. 3 A depiction of the parameter in Eq. 7. How far a vehicle can travel based on its endurance (T) and cruise velocity $\left(V_{c r}\right)$ compared to domain size $(L)$.

The next parameter is the turning ratio and is defined as:

$$
\frac{R_{t}}{L}
$$

It is a ratio of the vehicles' turning radii to the domain size. The turning radius is defined in Eq. 9. A graphic of this parameter is shown in Fig. 4. To determine the effect this number had on vehicle performance, we varied this number from 0.025 to 0.225 to test a wide range of turning radii. A turning ratio of 0.025 allowed 40 turns to be completed in the domain while a turning ratio of 0.225 allowed less than 5 completed turns. This range allowed us to see the full effect turning ratio had on search performance.

$$
R_{t}=\frac{V_{s}^{2}}{g \tan \phi}
$$

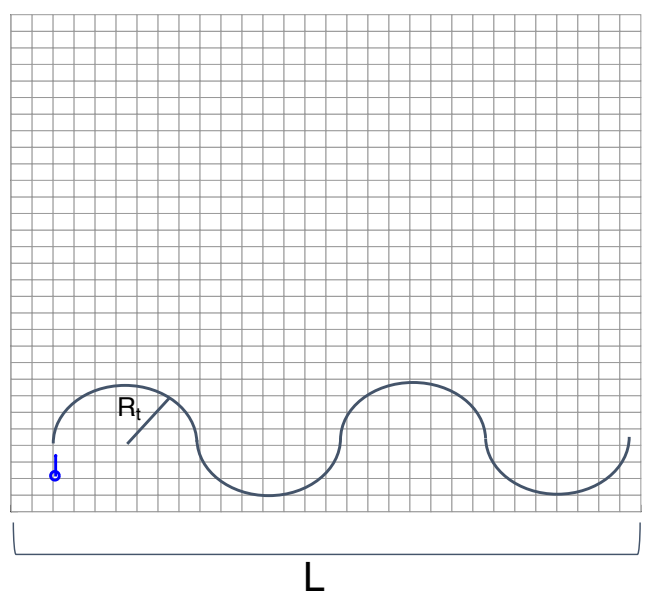

Fig. 4 A depiction of the parameter in Eq. 8. How many times a vehicle can turn with specified turn radius $\left(R_{t}\right)$ within the domain size $(L)$.

The next parameter is the communication ratio and is defined as:

$$
\frac{R_{c}}{R_{S}}
$$


It shows how far the vehicles can communicate compared to what the vehicles can sense. We kept this value constant at a value of one so we could examine the other vehicle properties.

The following parameter is the area ratio and is defined as:

$$
\frac{N_{v} R_{s}^{2} \pi}{L^{2}}
$$

This gives the ratio between the amount of area sensed by all vehicles, $N_{v}$, to the total area of the domain. For the analysis we varied this parameter from 0.1 to 0.9 .

The parameter:

$$
\frac{l_{c}}{L}
$$

represents the length of a grid cell to the length of the domain size, and the parameter:

$$
\frac{t}{T}
$$

is the duration of one time step, or the length of time before a vehicle changes state to the next roll angle, to the aircraft endurance. These parameters are not physically meaningful and are only artifacts of the discretization. Therefore, they were kept constant across the different studies.

\section{Reward Function}

We used gradient-based optimization by utilizing the MATLAB function fmincon, which maximizes or minimizes a specified reward function. The design variables were the planned roll angles which guided the vehicles' motion. The reward function used the MATLAB ODE45 solver to solve equations 4. 5. and 6 for the heading angle, north position, and east position of the vehicle after each roll angle. We then calculated the distance along the vehicle's entire planned path to every grid center. After tabulating these distances, we recorded the minimum distance to each cell from the vehicle over the entire path and updated the cell value according to the Gaussian distribution in Eq. 11. We summed the value across all cells for the final reward.

The total reward for the $j t h$ iteration is calculated as:

$$
J_{j}=\sum_{i=1}^{N_{g}} r_{i, j}
$$

The number of grid cells is represented as $N_{g}$ and $r_{i}$ represents the predicted value of that cell from the path in question, calculated in Eq. 1

We knew vehicles needed to plan future time steps to avoid obstacles and maximize reward. However, because computational expense scales with number of design variables, it is desirable to use fewer look ahead steps. At the same time, minimizing the number of look ahead steps limits the vehicle's perception of potential mission costs. This can gravely impact mission completion. We used six look ahead steps because we found increasing beyond this number resulted in no further performance benefit. Additionally, because gradient based optimization can find local maximums, a multistart was used with three initial sets of starting roll angles. The vehicle could proceed left, straight, or right. The actual path that was taken was the path that achieved the largest maximum reward.

\section{Communication Cases}

To test how the amount of information shared affected performance, we developed four communication cases: a decentralized no communication case, a decentralized case in which current and past locations were shared, and both a decentralized and centralized case where the current, past, and future locations were shared. In the centralized case the path of all vehicles were optimized in unison. In the decentralized cases each vehicle optimized its own path based on available information. For these cases, we assumed there was perfect communication and every vehicle instantaneously communicated with the other vehicles. In addition, a linear time-based decay was added with the cells' value slowly decreasing over time. As a result, cells would require continuous surveillance. We averaged the performance metric in Eq. 2 across all time steps to determine how the vehicles performed at searching throughout the entire simulation. Since the performance was averaged over the entire search mission and there was a time-based decay, the performance values were significantly lower than the non-dimensional results. 
The first simulation consisted of no communication, where vehicles knew nothing about the other vehicles. Therefore, we optimized each vehicle's path individually based only on its previous location history. For the second simulation, we optimized vehicles' paths based on the current state of the search area. This represented a case in which vehicles communicated past and current location with one another. In the third simulation, we assumed there was full communication of past, current, and future paths. Thus, we optimized vehicles' paths based on a grid that also accounted for the future planned location of each vehicle. We ran this case with both a centralized and decentralized approach. We assessed the trials according to the performance, given by Eq. 2, averaged over the entire simulation.

We kept the vehicle properties constant among all four cases so the only factor that was changing from case to case was the amount of communication. We set the endurance ratio to 10 , the turning ratio to 0.015 , and the area ratio to 0.68 .

\section{Results}

Two types of studies are explored in this section. The first considers the effect of the primary nondimensional parameters across a wide range of variation. The goal is to understand what parameters have the strongest effect on performance, and what ranges of parameters are necessary to achieve a specified level of performance. Vehicles in this scenario communicate past, present, and future positions within their specified communication radius, using a decentralized approach.

The second study explores the effect of communication. Four levels of communication are considered, with communication occurring across the entire domain (infinite communication radius). The four levels are: no communication, sharing past and current positions, sharing past, current, and future positions, and sharing past, current, and future positions with centralized planning.

\section{A. Non-dimensional Analysis}

There were four primary non-dimensional parameters of interest as discussed previously in Sec. II.B. We kept the communication ratio constant at a value of one to reduce the number of necessary optimizations. Therefore, we varied three parameters across a range of combinations. We ran simulations across five different area ratios, five different endurance ratios, and five different turning ratios for a total of 125 optimizations. Figs. 5, 6, and 7 all use the same data gathered from these optimizations. The only difference between the plots is what ratio is used on the $\mathrm{x}$-axis and what parameter is changed from plot to plot. 


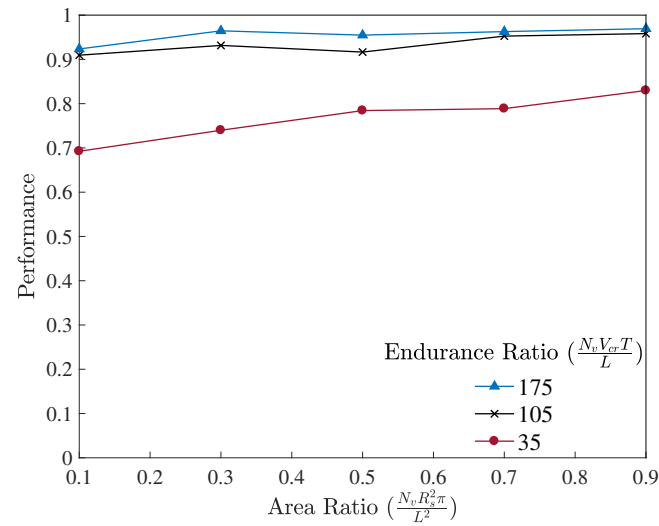

(a) turning ratio $=\mathbf{0 . 0 2 5}$

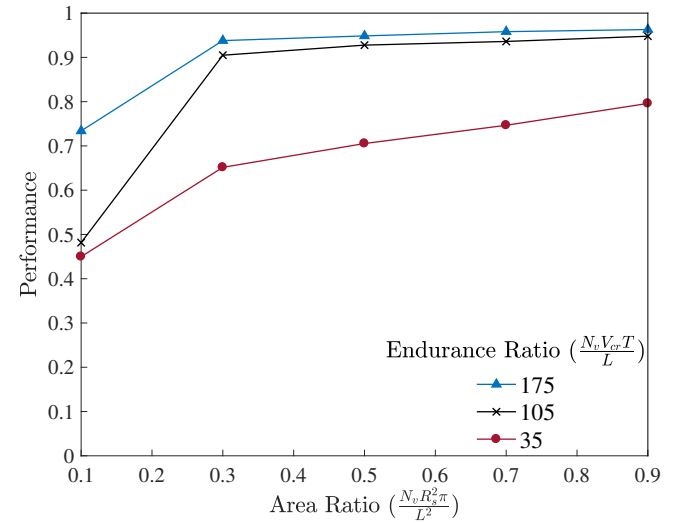

(b) turning ratio $=\mathbf{0 . 1 2 5}$

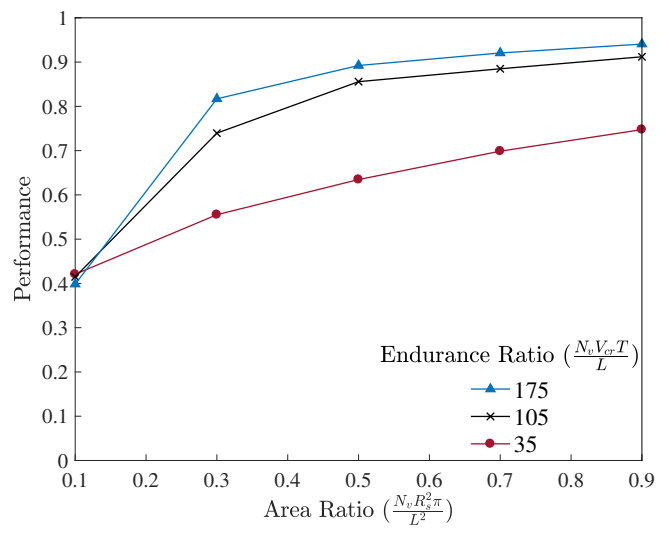

(c) turning ratio $=\mathbf{0 . 2 2 5}$

Fig. 5 Each figure shows the performance verses area ratio. Each line represents a different endurance ratio and each different plot corresponds to a different turning ratio.

Each plot in Fig. 5 shows the performance verses area ratio. Each line represents a different endurance ratio and each plot is plotted at a different turning ratio. As shown from Fig. 5a where the turning ratio is 0.025 , when the turning radius is small compared to the domain size, there is not a significant increase in performance when increasing the area ratio. In fact, at high endurance ratios there is no improvement for increasing the area ratio. This suggests that when the chosen vehicles are agile and can perform tight turns, the amount of area the vehicles are capable of sensing at one time step is not as important. In terms of the reward function, the vehicles have more freedom to turn to explore cells that have not been searched. As seen from Figs. $5 \mathrm{~b}$ and $5 \mathrm{c}$, when the turning ratio is large, the area ratio becomes increasingly more important. Since a vehicle is unable to make tight turns, the area sensed by the vehicles needs to be larger. A vehicle needs to make up for its lack of agility by sensing more area. This way the vehicles are able to see more of the grid at each time step while performing more gradual turns. In addition, all figures show a smaller difference in performance between endurance factors of 15 and 25. This conveys that the search missions do not benefit from increasing the vehicles' endurance after a certain point (further discussed after Fig. 6below). The increase in endurance leads to the vehicles' exploring area that has already been seen. 


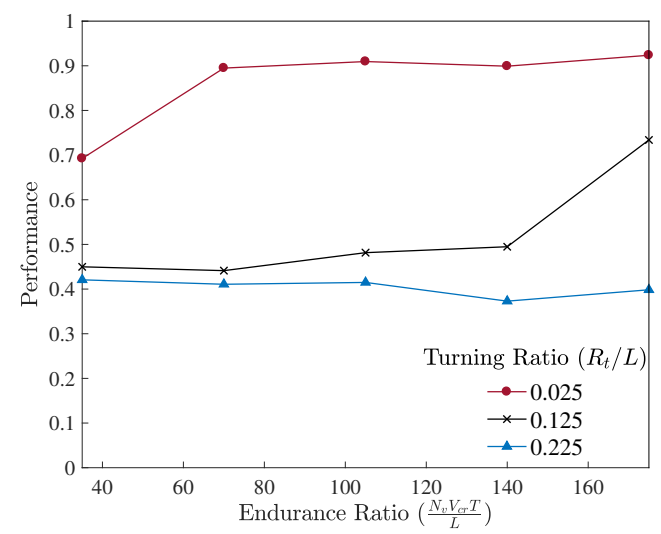

(a) area ratio $=0.1$

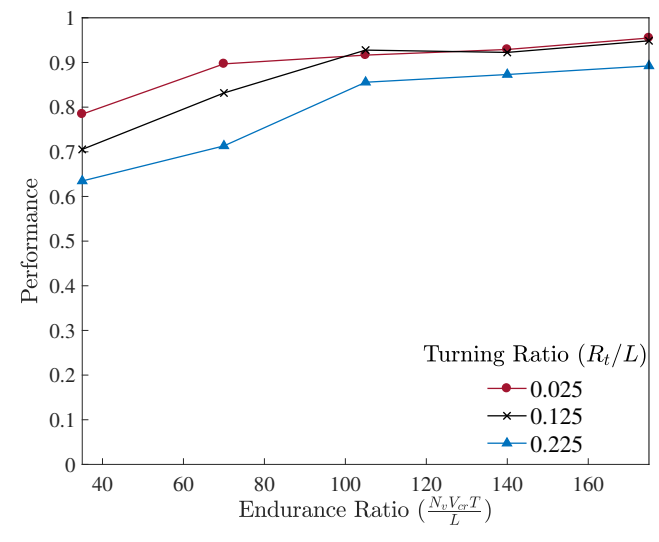

(b) area ratio $=0.5$

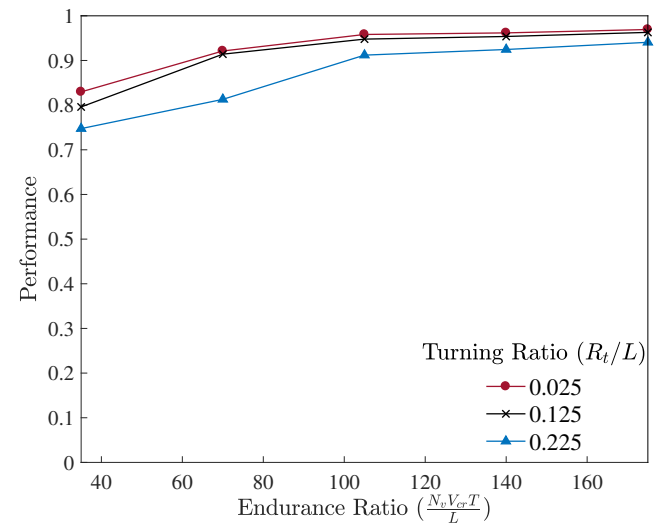

(c) area ratio $=0.9$

Fig. 6 Each figure shows the performance verses endurance ratio. Each line represents a different turning ratio and each different plot corresponds to a different area ratio.

In Fig. 6 the performance is plotted across the endurance ratio where the lines are plotted at different turning ratios. Each plot has a different area ratio. It can been seen from Fig. 6a that when the area ratio is small, the turning ratio significantly alters the mission performance outcome. The figure shows that even when the area ratio is small as 0.1 , the vehicles can coordinate and search $90 \%$ of the grid if the turning ratio is 0.025 and the endurance ratio is 70 . This shows that as long as the vehicles have a small turning ratio the majority of the grid is searched. However, Figs. 6b and $6 \mathrm{c}$ demonstrate that even if the turning ratio is large, high performance can still be achieved as long as the vehicles can sense more area. In addition, Fig. 6c shows that once the area ratio is high, a small turning ratio provides no further benefit to performance.

It appears that the increase in performance from the endurance ratio plateaus at a value of 105 for Figs. $6 \mathrm{~b}$ and $6 \mathrm{c}$ From Fig. 6a it appears that the performance is more susceptible to changing the endurance factor. When the turning ratio was 0.025 , the performance plateaued after an endurance ratio of 70 . For the turning ratio of 0.125 , the performance just started benefiting from an endurance ratio of 175 . For the turning ratio of 0.225 , there has not yet been an increase in performance and the endurance needs to be further increased. This demonstrates that when both the turning ratio and area ratio are unsuitable for the domain, the endurance ratio needs to be very high to account for the poor maneuverability and sensing properties of the vehicles. 


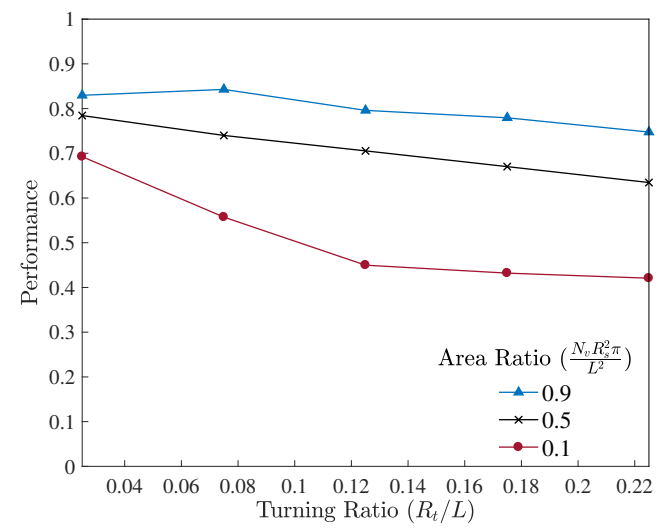

(a) endurance ratio $=35$

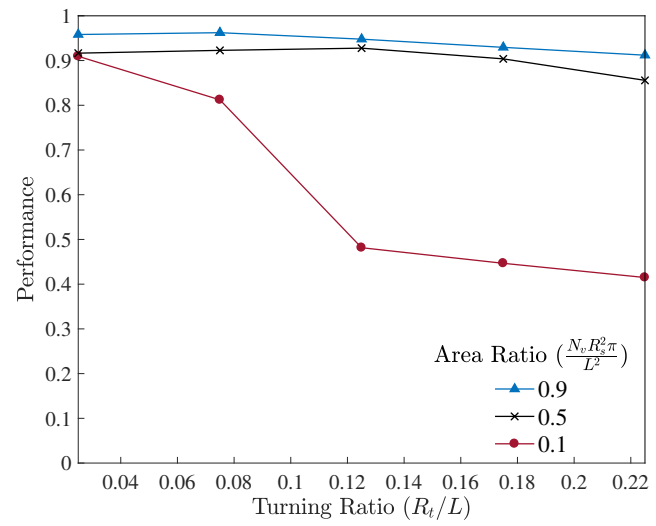

(b) endurance ratio $=105$

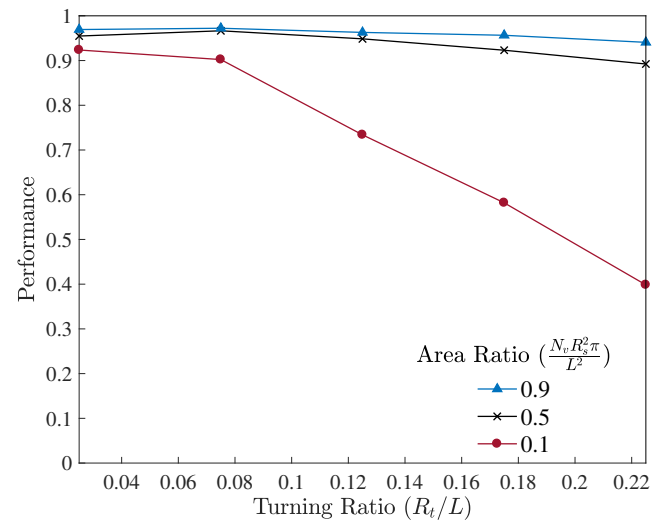

(c) endurance ratio $=175$

Fig. 7 Each figure shows the performance verses turning ratio. Each line represents a different area ratio and each different plot corresponds to a different endurance ratio.

In Fig. 7 , the performance ratio is plotted across the turning ratio with each line corresponding to a different area ratio. Each figure has a different endurance ratio. As seen from Fig. 7 , for good performance the turning ratio needs to be small enough based on the given area ratio and endurance. Figures $7 \mathrm{~b}$ and $7 \mathrm{c}$ show that with the combination of a high endurance and small turning ratio, there is little to no benefit associated with increasing the area ratio. However, the turning radius does significantly affect the performance when the area ratio is low. Additionally, Fig. 7c shows that at an area ratio of 0.1 , the performance plateaus when the turning ratio is 0.08 . At this same area ratio in Fig. $7 \mathrm{~b}$, the performance is just beginning to plateau whereas in Fig. 7a, the performance has yet stabilize. Therefore, the turning ratio needed to maximize search performance becomes increasingly smaller as the area ratio and endurance ratio decrease.

\section{B. Effect of Communication}

For swarm mission completion, communication is required, however, the amount of communication is unknown. We changed the amount of communication in each simulation, then compared the average search performance of the swarm. We ran a test with no communication to determine a baseline for performance without any communication. Additionally, we created a centralized case to know how the communication compares with perfect in unison communication. The performance of all cases is provided in Table 1. Since the difference between communication strategies is difficult to see in static plots, we provided a video that shows the trajectories of all four communication cases: https://youtu.be/GkR6KVicwPI

For the no communication case, we optimized each vehicle's path as if it was the only vehicle performing the mission. For each vehicle's optimization, we set the grid to replicate only what each respective vehicle sensed. We 
only changed the grid cell values based on the location of each individual vehicle. Vehicles primarily proceeding in a straight line except when approaching a grid boundary. Since the vehicles did not communicate, each vehicle did not know which cells were previously explored. In addition, since the domain was large compared to the vehicles' sensing radius, the potential reward was only decreased when a vehicle approached a grid boundary. As a result, the vehicles did not come close to the edge, so reward could be maximized without the search radius being outside of the domain. In addition, there was vehicle clustering with some cells not being searched at all.

In the second case, it was assumed that there was perfect communication of the vehicles' past and current locations within their sensing radius. For each optimization, we optimized the vehicles' paths based on grid values that reflected the motion of every other vehicle. We used a decentralized approach in which vehicles optimized paths based on the previous step of all other vehicles to replicate the behavior of the simultaneous movement of vehicles. The vehicles remained more dispersed, however, there was still vehicle clustering where vehicles searched the same cell.

In the third case, in addition to the communication of the current and past location, vehicles also communicated where they were planning on traveling. Each vehicle optimized based on grid values that were reflective of each vehicle's future planned path. For this case, we also used decentralized path planning. Again, we optimized each path based on the planned path from the previous time step of all other vehicles. This case had the most equal distribution of vehicles out of the decentralized cases.

In the final case case, a centralized approach was used where we used one function to optimize the path of all vehicles. Thus, this case represented in-sync decision making. This approach was very costly compared to the other cases, however, vehicles remained dispersed and never searched the same cell at the same time. However, Table 1 shows only a $1.7 \%$ increase from the decentralized to centralized case.

\begin{tabular}{|c|c|c|c|}
\hline No Communication & Current Location & Full Decentralized & Full Centralized \\
\hline $35.9 \%$ & $42.2 \%$ & $44.5 \%$ & $46.2 \%$ \\
\hline
\end{tabular}

Table 1 Performance percentages of different communication cases

Sharing only the vehicles' current locations improved performance by $6.3 \%$. The type of communication varied performance by $2.3 \%$ and the centralized case improved performance by $1.7 \%$. This shows that any type of communication improves performance. However, the type of communication has less impact on the search performance. In addition, this shows centralized communication is, for the most part, not worth the large increase in computational cost.

\section{Conclusions}

Our goal was to find how different types of communication and vehicle parameters affected the search performance for a swarm of UAVs. Unlike other research, instead of comparing different optimization algorithms we analyzed communication alone. Additionally, we did not set the vehicle properties as constraints, but we varied these properties to determine the trends in search performance for different properties. We used non-dimensionalization so our results could be applied to a variety of scenarios.

These results convey that for maximum performance it is desirable to have a low turning ratio and high endurance and area ratios. However, if the simulation has a low area ratio, high performance can still be achieved if the turning ratio is sufficiently small and the endurance ratio is sufficiently large. We determined that even if vehicles could only sense $10 \%$ of the total area at one time step, $92 \%$ of the area could be seen by using a vehicle with good endurance and maneuverability. On the other hand, if the turning ratio is high, this same performance of $92 \%$ can be achieved with high endurance and area ratios. Therefore, this shows there are multiple ways to achieve high search performance by changing different mission parameters.

The communication study showed that when vehicles communicated the current location and past history, performance improved by $6.3 \%$. When vehicles communicated the planned future location as well, performance improved by $8.6 \%$. When we used a centralized method in which vehicles used an in-sync decision making process, performance only increased by $1.7 \%$ and the optimizations were computationally expensive. Therefore, for the majority of search missions this small increase in performance is not worth the large computational cost.

Little work has been performed regarding high density search swarms. In the future, optimization methods should be explored with swarms consisting of hundreds to thousands of vehicles. This presents many challenges involving the large number of trajectory optimizations. Efficient methods would need to be devised to coordinate this many vehicles 
while trying to minimize the large computational cost. In addition, search missions can benefit from heterogeneous swarms. Adding diverse vehicles to a swarm can increase search performance by capitalizing on the different vehicle strengths. However, this would require the use of mission allocation methods to assign missions based on a variety of factors.

\section{Acknowledgements}

This work has been funded by the Center for Unmanned Aircraft Systems (C-UAS), a National Science Foundationsponsored industry/university cooperative research center (I/UCRC) under NSF Award No. IIP-1650547 along with significant contributions from C-UAS industry members. 


\section{References}

[1] Howard, A., Parker, L. E., and Sukhatme, G. S., "Experiments with a Large Heterogeneous Mobile Robot Team: Exploration, Mapping, Deployment, and Detection,” The International Journal of Robotics, Vol. 25, No. 5-6, 2006.

[2] Gade, S., and Joshi, A., "Heterogeneous UAV swarm system for target search in adversarial environment," Control Communication and Computing, Thiruvananthapuram, India, 2013.

[3] Jaimes, A., Kota, S., and Gomez, J., "An Approach to Surveillance an Area Using Swarm of Fixed Wing and Quad-rotor Unmanned Aerial Vehicles UAV(s)," System of Systems Engineering, edited by IEEE, 2008.

[4] Zhang, B., Liu, W., Mao, Z., Liu, J., and Shen, L., "Cooperative and Geometric Learning Algorithm (CGLA) for path planning of UAVs with limited information," Automatica, Vol. 50, No. 3, 2014, pp. 809-820.

[5] Deng, Q., Yu, J., and Wang, N., "Cooperative Task Assignment of Multiple Heterogeneous Unmanned Aerial Vehicles Using a Modified Genetic Algorithm with Multi-type Genes,” Chinese Journal of Aeronautics, Vol. 26, No. 5, 2013, pp. $1238-1250$.

[6] Huang, Y., Xu, J., Qiu, L., and Zhang, R., “Cognitive UAV Communication via Joint Trajectory and Power Control,” 2018.

[7] Alshbatat, A. I., and Dong, L., "Performance Analysis of Mobile Ad Hoc Unmanned Aerial Vehicle Communication Networks with Directional Antennas," International Journal of Aerospace Engineering, 2010, p. 14.

[8] Waharte, S., and Trigoni, N., "Supporting Search and Rescue Operations with UAVS," Emerging Security Technologies, 2010.

[9] Krieger, M. J., and Billeter, J.-B., "The call of duty: Self-organised task allocation in a population of up to twelve mobile robots," Robotics and Autonomous Systems, Vol. 30, No. 1-2, 2000, pp. 65-84.

[10] Arkin, R. C., Balch, T., and Nitz, E., "Communication of Behavioral State in Multi-agent Retrieval Tasks," IEEE ICRA, Vol. 3, 1993, pp. 588-594.

[11] Trianni, V., and Dorigo, M., "Self-organisation and communication in groups of simulated and physical robots," Biological Cybernetics, Vol. 95, No. 3, 2006.

[12] Sujit, P., and Ghose, D., "Search using multiple UAVs with flight time constraints," IEEE Transactions on Aerospace and Electronic Systems, Vol. 40, No. 2, 2004.

[13] Blasi, L., Barbato, S., and Mattei, M., "A particle swarm approach for flight path optimiation in a constrained environment," Aerospace Science and Technology, Vol. 26, No. 1, 2013, pp. 128-137.

[14] Nigma, N., Bieniawski, S., Kroo, I., and Vian, J., "Control of Multiple UAVs for Persistent Surveillance: Algorithm and Flight Test Results," IEEE Transactions on Control Systems Technology, Vol. 20, No. 5, 2012. 